\title{
Género, formación profesional e inserción laboral
}

\section{Gender, Professional Training and Labour Insertion}

\section{RESUMEN}

Es cierto que es preciso actualizar los estudios de formación profesional, mejorar la empleabilidad, dar respuestas a las necesidades del mercado de trabajo, pero, sobre todo, hay que tener en cuenta un aspecto fundamental como es la igualdad de género. En este sentido, es prioritario abordar los problemas que conlleva la brecha de género en el acceso a los estudios de formación profesional. Por ello, en este trabajo se analiza de forma detallada la presencia de las mujeres en estos estudios, las razones que determinan la elección de unos u otros, la interdependencia entre los estudios cursados y la inserción laboral, así como los efectos negativos para las mismas a la hora de acceder al mercado de trabajo, pese a los avances en materia de igualdad de oportunidades entre hombres y mujeres, y, por último, las soluciones adoptadas y las posibles propuestas al respecto.

Palabras clave: género, estereotipos, socialización, formación profesional, inserción laboral.

\begin{abstract}
We need to update the studies of professional training, improve employability and respond to the needs of the labour market, but, above all, we need to take into account a fundamental aspect such as gender equality. In this respect, priority must be given to tackling the problems that gender gap implies in access to professional training. Thus, this paper analyses in detail the presence of women in these studies, the reasons that determine the choice for one or other studies, the interdependence between their studies and their labour insertion, the negative effects on women in the access to the labour market, despite the progress in equal opportunities for men and women, and finally the adopted solutions and possible proposals in this regard
\end{abstract}

Keywords: gender, stereotypes, socialization, professional training, labour insertion.

\section{SUMARIO}

1.- Introducción. 2.- La relevancia de los estudios de formación profesional y la necesidad de introducir modificaciones. 3.- El acceso a los estudios de formación profesional y la brecha de género. 3.1.- El principio de igualdad y su proyección en el acceso a los estudios de formación profesional. 3.2.- De la presencia paritaria a la brecha de género en las vocaciones. 3.3.- La socialización de género y su intervención en la elección de los estudios de formación profesional. 4.- Conexión entre inserción laboral y formación profesional. 4.1.- Breve referencia a la igualdad de oportunidades entre hombres y mujeres en el acceso al empleo. 4.2.- La interdependencia entre estudios cursados e inserción laboral. 4.3.- Los efectos perjudiciales de la socialización de género para las estudiantes de formación profesional. 5.- Soluciones y propuestas. -Bibliografía. 


\section{1.- Introducción}

A pesar de que los estudios de formación profesional (FP) han venido adquiriendo relevancia de forma progresiva y se ha ido incrementando el número de ofertas de empleo para quienes realizan estos estudios, es preciso plantearse la necesidad de alcanzar la igualdad de género, en concreto, con respecto a la inserción en el mercado de trabajo de las mujeres que han cursado estos estudios y a los términos en los que esta tiene lugar; sobre todo, si se tiene en cuenta el problema de la segregación ocupacional por género que se produce y que las sitúa en una posición de desventaja significativa en el mercado laboral, pese al importante papel que tienen en el mismo (Bochholz y Grunow, 2006). Es cierto que La Ley Orgánica 3/2007, de 22 de marzo, para la igualdad efectiva de mujeres y hombres (LOI) supuso un claro avance en la materia, y que debería de haber contribuido a eliminar esa situación, sin embargo las desigualdades de género han venido persistiendo hasta la actualidad en el mercado laboral; lo que se ha reconocido por el propio legislador en el Real Decreto-Ley 6/2019, de 1 de marzo, de medidas urgentes para garantía de la igualdad de trato y de oportunidades entre mujeres y hombres en el empleo y la ocupación, en el que se hace referencia a la infrarrepresentación femenina en determinadas profesiones, entre las que se pueden incluir algunas para las que es preciso haber cursado estudios de FP. Una norma en la que se han establecido medidas novedosas, pero cuyo contenido resulta insuficiente ya que no se cumplen las expectativas que se contemplan en su preámbulo (Ballester, 2019: 15, 22-23; Pastor, 2019: 189).

Partiendo de lo señalado, este trabajo se centra en una cuestión que hasta el momento se ha tratado de forma limitada, en concreto, las transiciones formativolaborales de quienes cursan estudios de FP, analizándolas desde la igualdad de género, para determinar qué factores influyen en la inserción laboral de las mujeres que cursan dichos estudios. Lo que se aborda de forma transversal, sin centrarse única y exclusivamente en el momento de acceso de este colectivo al mercado de trabajo, sino situándose, previamente, en el de la elección de estos estudios, con el fin de constatar qué factores intervienen en la misma y las consecuencias que después puede tener en cuanto a la inserción laboral, para determinar hasta qué punto puede influir en la citada segregación laboral, y si puede ser un elemento adicional a tener en cuenta junto a las medidas que se puedan adoptar desde el punto de vista laboral.

En cuanto a la metodología seguida cabe señalar que se ha realizado un estudio de la materia en el que se abordan aspectos educativos, laborales y, a su vez, la relación entre ambos, y, para ello, se ha buscado apoyo en las aportaciones disponibles en las distintas fuentes sobre la misma, teniendo en cuenta las conexiones que se ponen de manifiesto en el trabajo, como sucede con la realidad estrictamente normativa en materia de igualdad, en los ámbitos laboral y educativo, que, a su vez, conecta con la orientación de la FP al desarrollo personal, al ejercicio del derecho al trabajo y a la elección de profesión u oficio. Si bien, puesto que se ha planteado si la socialización de género puede tener influencia en las elecciones que se realizan y, 
por tanto, si puede ser un factor que ha de considerarse en cuanto a la inserción en el mercado de trabajo, ha sido necesario acudir a los análisis realizados en este ámbito para determinar cómo opera en este caso y deducir las posibles repercusiones. Junto a lo anterior, se han consultado encuestas y datos estadísticos teniendo en cuenta diferentes fuentes de información, entre las que cabe destacar, el Ministerio de Educación y Formación Profesional (MEyFP) y el Instituto Nacional de Estadística (INE). Lo que ha permitido efectuar un análisis a partir de datos desagregados por género, para variables relevantes para el objeto de estudio, con el fin de realizar un diagnóstico de la situación; según los casos, se ha partido de datos en cifras absolutas y/o porcentuales, que permiten identificar situaciones de desigualdad y medir la posible brecha de género que se puede producir. Es importante señalar que el análisis empírico que se realiza se justifica por el hecho de que pueda ser aplicable en el ámbito laboral; razón por la que, necesariamente, se aborda desde la generalidad y la abstracción con la que se construyen las regulaciones normativas.

\section{2.- La relevancia de los estudios de formación profesional y la necesidad de in- troducir modificaciones}

A pesar de que los estudios de FP se han venido considerando como una especie de segunda opción, en la actualidad, también se presentan como una alternativa que puede facilitar la inserción laboral. De hecho, si se consultan las estadísticas del MEyFP, curso 2017/2018, se puede constatar que el alumnado que realiza estudios de FP se ha ido incrementando progresivamente, sobre todo si se compara el número de alumnos en el curso 2012/2013 con respecto al del curso 2017/2018; en concreto, se ha producido un aumento del $23,3 \%$ en su conjunto. En este sentido, cabe destacar la implantación de la FP Básica (FPB), con una cifra inicial de 39.867 alumnos en el curso 2014/2015 hasta llegar a 72.180 alumnos en el curso 2017/2018, y, sobre todo, el aumento de un 21,4\% de estudiantes de FP de Grado Superior (FPGS), y un 73,4\% del régimen a distancia (MEyFP, Estadísticas del alumnado de FP). Cifras que se han seguido incrementando en el curso 2018/2019, un 2,5\% más en la FPB, con 74.009 alumnos; un 1,9\% adicional en la FP de Grado Medio (FPGM), con 350.820 alumnos y un 3,8\% más en el caso de la FPGS, con un total de 413.935 estudiantes (MEyFP, 2019b: 4).

A su vez, se ha ido elevando el número de ofertas de empleo para quienes han realizado estudios de FP hasta llegar en el año 2018 al 42,4\% del total de la oferta de empleo en España. De estas, el 24,44\% corresponde a las titulaciones de FPGS, las más demandadas, a pesar de haber disminuido el porcentaje con respecto al año anterior (25,48\%), y el 17,82\% a ofertas de empleo de titulaciones de FPGM, un porcentaje más elevado que el del año 2017 (14,84\%). En todo caso, la oferta ha sido mayor que la del año anterior, lo que obedece a factores como la especialización, la accesibilidad y su carácter práctico (Adecco Group, 2018: 17 y 118).

$\mathrm{Al}$ respecto, hay que destacar la importancia que tiene en los currículos de los distintos ciclos formativos el módulo profesional de Formación en Centros de Trabajo, con el que se potencia la formación de carácter práctico; lo que sucede en 
mayor medida en el caso de la modalidad de FP Dual (FPD) ${ }^{2}$, regulada por el Real Decreto 1529/2012, de 8 de noviembre, por el que se desarrolla el contrato para la formación y el aprendizaje y se establecen las bases de la FPD (RD 1529/2012), en la que se combina la formación en el centro educativo y en el de trabajo, $\mathrm{y}$, por tanto, el aprendizaje teórico y práctico. Una formación basada en la colaboración y la participación de las empresas de forma más activa en el proceso de formación del estudiante; lo que permite que se vincule y se adapte a las demandas y necesidades de las mismas, y puede facilitar la transición del sistema educativo al mercado de trabajo, como sucede en países como Alemania, Austria, Dinamarca, etc. Si bien, para ello es necesario que exista una verdadera colaboración entre las empresas y los centros de FP, y que la cualificación que adquieren los estudiantes sea de calidad; lo que requiere la implicación de centros y empresas. En España esta formación adolece de importantes «deficiencias político-estructurales» (Alemán, 2018) que será preciso solventar para conseguir la finalidad que persigue la misma. A lo largo de los últimos cursos académicos se ha avanzado en la implantación de proyectos de FPD en distintas Comunidades Autónomas, pero con modelos diferentes. Sería necesario establecer un marco común adaptado en el que se tuviera en cuenta el hecho de que en España predomina la pequeña empresa; lo que no hace posible implantar de forma directa los modelos de formación dual que han funcionado en otros países.

En todo caso, con carácter general, es preciso introducir modificaciones en materia de FP para seguir mejorando la empleabilidad y dar respuesta a las necesidades del mercado de trabajo; «un mercado en el que 8 de cada 10 empresas afirman haber tenido dificultades para cubrir determinadas vacantes en el ejercicio 2018» (Adecco Group, 2018: 9). Es más, los estudios del Centro Europeo para el Desarrollo de la Formación Profesional ${ }^{3}$ señalan que se va a producir un incremento en la demanda de perfiles de FP. Se prevé que en España, en el año 2025, el 49\% de los puestos de trabajo se vinculen a personas con un nivel de cualificación medio, es decir, con estudios de FP. Sin embargo, a pesar del incremento en el número de alumnos matriculados en estos estudios, en España el número de jóvenes de entre 15 y 19 años matriculados en los mismos es bastante reducido (18,7\%) si se compara con otros países de la OCDE (31\%, MEyFP, 2019a:19); produciéndose un desequilibrio entre el nivel de cualificación de la población y las demandas del mercado de trabajo.

En este sentido, cabe destacar el I Plan Estratégico de Formación Profesional del Sistema Educativo 2019-2022 (PEFP), que se deriva de la Agenda 2030 y sus objetivos para el Desarrollo Sostenible, como son, entre otros, garantizar una educación inclusiva y equitativa de calidad; promover el aprendizaje permanente; lograr la igualdad de género y empoderar a todas las mujeres y niñas; promover el crecimiento económico sostenido, inclusivo y sostenible, el empleo pleno y productivo,

2 Vid. Arts. 6 y 11.3 de la Ley Orgánica 5/2002, de 19 de junio, de las Cualificaciones y de la Formación Profesional, con respecto a la colaboración de las empresas en el desarrollo del Sistema Nacional de Cualificaciones y Formación Profesional (LOCFP).

3 Vid. https://www.cedefop.europa.eu/es/translated-content/es?page=1 
el trabajo decente para todos, y la transición ecológica socialmente justa y fomentar la innovación. Objetivos que implican la introducción de cambios en materia de FP. Así, el PEFP pretende actualizar estos estudios, mejorar la empleabilidad y ofrecer una respuesta a las citadas necesidades del mercado de trabajo; además de modificar la normativa relativa a las enseñanzas de FP, en concreto, el RD 1529/2012 en cuanto a la FPD, y renovar el Catálogo de títulos de FP, incluyendo nuevas formaciones, en particular, perfiles profesionales vinculados con la digitalización, la industria 4.0, el big data, la inteligencia artificial, la economía circular y la transición ecológica. Sin olvidar que, en esa necesaria modificación de los estudios de FP, es preciso considerar un aspecto fundamental como es la igualdad de género.

\section{3.- El acceso a los estudios de formación profesional y la brecha de género}

En el PEFP se indica que «en pleno siglo XXI no hay ninguna razón para que determinadas profesiones estén ocupadas mayoritariamente por mujeres y otras por hombres», por ello, es importante analizar por qué se produce esta situación.

\section{1.- El principio de igualdad y su proyección en el acceso a los estudios de for- mación profesional}

La igualdad se configura como un principio que está presente en la Ley Orgánica 2/2006, de 3 de mayo de Educación (LOE). En concreto, en el art. 1 LOE, en el que se contemplan los principios en los que se inspira el sistema educativo español, cabe destacar cómo el legislador hace referencia expresa a «la igualdad de derechos y oportunidades, también entre hombres y mujeres, que ayuden a superar cualquier discriminación» (apartado b); así como al «desarrollo de la igualdad de derechos, deberes y oportunidades» y al «fomento de la igualdad efectiva de mujeres y hombres» (apartado 1). Principios por los que la inspección educativa deberá «velar» por su «cumplimiento y aplicación» (art. 151.e) LOE).

Por su parte, el art. 2.1 b) LOE, entre los fines de la educación, contempla precisamente «la educación [...] en la igualdad de derechos y oportunidades entre hombres y mujeres». Lo que, a su vez, se proyecta en la norma a partir del establecimiento de diferentes obligaciones, como la necesidad de que el Consejo Escolar designe «una persona que impulse medidas educativas que fomenten la igualdad real y efectiva entre hombres y mujeres» (art. 126.2 LOE); de que los libros de texto y demás materiales curriculares reflejen y fomenten «el respeto» a la citada igualdad (DA $\left.4^{\mathrm{a}} .2 \mathrm{LOE}\right)$; y en forma de objetivos de las enseñanzas que ofrece el sistema educativo. En este sentido, se establece cómo las distintas etapas deben contribuir a desarrollar una serie de capacidades de los alumnos y de las alumnas que les van a permitir, en la educación primaria, «conocer, comprender y respetar [...] la igualdad de derechos y oportunidades de hombres y mujeres» (art. 17.d) LOE); y, en la educación secundaria obligatoria (ESO), «valorar y respetar la diferencia de sexos y la igualdad de derechos y oportunidades», y «rechazar los estereotipos que supongan discriminación entre hombres y mujeres» (art. 23.c) LOE). Lo que es relevante 
en su trayectoria académica y profesional. De esta forma, antes de cursar enseñanzas postobligatorias, deben de haber recibido una formación y unos valores en los que está presente la igualdad entre hombres y mujeres, que también se refleja en estas enseñanzas, ya que el art. 40.1 e) LOE establece, entre los objetivos relativos a las enseñanzas de FP, que contribuirá a que el alumnado consiga los resultados de aprendizaje que le permitan «fomentar la igualdad efectiva de oportunidades entre hombres y mujeres».

De hecho, cabe destacar que la igualdad de oportunidades de la que disponen hombres y mujeres en el acceso a los estudios de FP se configura como un principio que inspira el Sistema Nacional de Cualificaciones y FP. El art. 2.3 LOCFP establece «el acceso, en condiciones de igualdad de todos los ciudadanos, a las diferentes modalidades de la formación profesional», y que esta «estará orientada tanto al desarrollo personal y al ejercicio del derecho al trabajo como a la libre elección de profesión u oficio y a la satisfacción de las necesidades del sistema productivo y del empleo a lo largo de toda la vida». Así, es la libertad de elección de los ciudadanos de su trayectoria académica en condiciones de igualdad entre hombres y mujeres, lo que, a su vez, les va a permitir elegir una u otra profesión; sobre todo porque los distintos estudios y las diferentes profesiones y oficios son igualmente aptos para ser desempeñadas por unos y otras (Sáinz y Meneses, 2018: 25). La norma no configura estudios y profesiones con género, de hecho, el art. 3.2 RD 1147/2011, de 29 de julio, en el que se establece la ordenación general de la formación profesional del sistema educativo, señala que «la formación profesional también fomentará la igualdad efectiva de oportunidades para todos, con especial atención a la igualdad entre hombres y mujeres».

\section{2.- De la presencia paritaria a la brecha de género en las vocaciones}

Si se parte de los últimos datos que existen en cuanto al alumnado de FP, se observa que en el curso 2017/2018 (Tabla I) el número de estudiantes ascendió a 815.354, si bien, las mujeres tienen una presencia inferior a la de los hombres, que se va incrementando en los niveles superiores. Así, en la FPB las mujeres representan el 29,2\%; en la FPGM, el 43,3\%; y en la FPGS alcanzan el 47,4\%. Lo que supone que, a pesar de que hay un mayor número de hombres, en estos últimos casos existe un equilibrio en cuanto a la presencia de hombres y mujeres en este tipo de estudios; en mayor o menor medida, existe paridad.

\begin{tabular}{|c|c|c|}
\hline \multicolumn{3}{|c|}{$\begin{array}{l}\text { Tabla I. Porcentaje de hombres y mujeres que cursaron estudios de formación } \\
\text { profesional (curso 2017/2018) }\end{array}$} \\
\hline Estudios & Hombres & Mujeres \\
\hline Formación Profesional Básica (FPB) & $70,8 \%$ & $29,2 \%$ \\
\hline $\begin{array}{l}\text { Formación Profesional de Grado Me- } \\
\text { dio (FPGM) }\end{array}$ & $57,8 \%$ & $42,2 \%$ \\
\hline
\end{tabular}




\begin{tabular}{|lll|}
\hline $\begin{array}{l}\text { Formación Profesional de Grado Supe- } \\
\text { rior (FPGS) }\end{array}$ & $44,6 \%$ \\
\hline $\begin{array}{l}\text { Formación Profesional a distancia } \\
\text { (FPaD) }\end{array}$ & $40 \%$ & $60 \%$ \\
\hline FPB + FPGM + FPGS + FPaD & $55,8 \%$ & $44,2 \%$ \\
\hline
\end{tabular}

Fuente: Datos y cifras. MEyFP Curso 2018/2019. Datos referidos al curso 2017/2018.

En términos generales, no existe una feminización o una masculinización de las enseñanzas de formación profesional consideradas en su conjunto. Sin embargo, es muy llamativo que gran parte de las estudiantes opten por determinados estudios (Tabla II), concentrándose en unas familias profesionales concretas:

\begin{tabular}{|c|c|c|c|c|c|c|}
\hline \multicolumn{7}{|c|}{$\begin{array}{l}\text { Tabla II. Estudios de Formación Profesional, alumnos, porcentaje de mujeres por } \\
\text { familias profesionales y tipo de formación (curso } 2017 / 2018 \text { ) }\end{array}$} \\
\hline \multirow{2}{*}{$\begin{array}{c}\text { Familias profe- } \\
\text { sionales }\end{array}$} & \multicolumn{2}{|c|}{ FP Básica } & \multicolumn{2}{|c|}{ FP Grado Medio } & \multicolumn{2}{|c|}{ FP Grado Superior } \\
\hline & Total & $\%$ Mujeres & Total & $\%$ Mujeres & Total & $\%$ Mujeres \\
\hline TOTAL & 69.582 & 29,2 & 343.920 & 43,3 & 372.937 & 47,3 \\
\hline $\begin{array}{c}\text { Actividades } \\
\text { físicas y depor- } \\
\text { tivas } \\
\end{array}$ & -- & .. & 9,737 & 20,6 & 21.042 & 20,2 \\
\hline $\begin{array}{c}\text { Administración } \\
\text { y Gestión }\end{array}$ & 12.410 & 50,7 & 50.536 & 60,5 & 56.102 & 63,3 \\
\hline Agraria & 4.099 & 19,7 & 7.229 & 14,7 & 6.415 & 19,3 \\
\hline Artes Gráficas & 493 & 42,2 & 3.342 & 35,3 & 2.051 & 47,3 \\
\hline $\begin{array}{l}\text { Artes y Arte- } \\
\text { sanía }\end{array}$ & -- & -- & -- & -- & 115 & 43,5 \\
\hline $\begin{array}{l}\text { Comercio y } \\
\text { Marketing }\end{array}$ & 3,962 & 53,7 & 15.123 & 53,8 & 23.309 & 48,2 \\
\hline
\end{tabular}




\begin{tabular}{|c|c|c|c|c|c|c|}
\hline $\begin{array}{c}\text { Edificación y } \\
\text { Obra Civil }\end{array}$ & 637 & 8,6 & 720 & 20,1 & 4.306 & 31,0 \\
\hline $\begin{array}{l}\text { Electricidad y } \\
\text { Electrónica }\end{array}$ & 9640 & 3,4 & 26.981 & 2,9 & 23.468 & 4,9 \\
\hline Energía y Agua & -- & -- & -- & -- & 2.244 & 9,8 \\
\hline $\begin{array}{l}\text { Fabricación } \\
\text { mecánica }\end{array}$ & 4.865 & 2,5 & 11.589 & 3,0 & 9.072 & 8,7 \\
\hline $\begin{array}{c}\text { Hostelería y } \\
\text { turismo }\end{array}$ & 6.426 & 38,4 & 19796 & 37,8 & 22.039 & 56,3 \\
\hline Imagen Personal & 5.860 & 84,7 & 18.741 & 91,2 & 7.295 & 94,3 \\
\hline $\begin{array}{l}\text { Imagen y So- } \\
\text { nido }\end{array}$ & -- & -- & 2.909 & 23,8 & 15.132 & 32,7 \\
\hline $\begin{array}{l}\text { Industrias Ali- } \\
\text { mentarias }\end{array}$ & 346 & 42,5 & 4.706 & 54,5 & 2.248 & 46,9 \\
\hline $\begin{array}{l}\text { Industrias Ex- } \\
\text { tractivas }\end{array}$ & -- & -- & 167 & 3,6 & -- & -- \\
\hline $\begin{array}{l}\text { Informática y } \\
\text { Comunicaciones }\end{array}$ & 10,980 & 17,8 & 31.078 & 8,0 & 44.473 & 11,7 \\
\hline $\begin{array}{l}\text { Instalación y } \\
\text { Mantenimiento }\end{array}$ & 381 & 3,4 & 11.609 & 2,1 & 12.208 & 14,4 \\
\hline $\begin{array}{l}\text { Madera, Mue- } \\
\text { bles y Corcho }\end{array}$ & 1.692 & 8,5 & 2.502 & 7,2 & 868 & 21,4 \\
\hline $\begin{array}{l}\text { Marítimo-Pes- } \\
\text { quera }\end{array}$ & 29 & 3,4 & 1.639 & 6,9 & 1.780 & 9,9 \\
\hline Química & -- & -- & 2.906 & 56,1 & 7.289 & 50,2 \\
\hline Sanidad & -- & -- & 72.119 & 72,3 & 46.206 & 73,7 \\
\hline $\begin{array}{l}\text { Seguridad y } \\
\text { Medio Am- } \\
\text { biente }\end{array}$ & -- & -- & 250 & 10,4 & 969 & 34,7 \\
\hline $\begin{array}{l}\text { Servicios So- } \\
\text { cioculturales y } \\
\text { a la Comuni- } \\
\text { dad }\end{array}$ & 235 & 78,3 & 21.347 & 85,9 & 56.836 & 87,3 \\
\hline
\end{tabular}




\begin{tabular}{|ccccccc|}
\hline $\begin{array}{c}\text { Textil, Confec- } \\
\text { ción y Piel }\end{array}$ & 444 & 54,3 & 1.274 & 82,3 & 1.521 & 86,2 \\
\hline $\begin{array}{c}\text { Transporte y } \\
\text { Mantenimiento }\end{array}$ & 6.960 & 1,9 & 27.511 & 2,4 & 10.904 & 2,8 \\
\hline $\begin{array}{c}\text { Vidrio y Cerá- } \\
\text { mica }\end{array}$ & 69 & 31,9 & 72 & 18,1 & 45 & 46,7 \\
\hline
\end{tabular}

Fuente: Datos y cifras. MEyFP Curso 2018/2019. Datos referidos al curso 2017 / 2018.

Como se puede apreciar, algunas especialidades están muy feminizadas, mientras que otras están masculinizadas; si bien, el número de familias que están masculinizadas es mucho más amplio. En concreto, los alumnos se distribuyen en quince familias profesionales (Actividades físicas y deportivas; Agraria; Edificación y Obra Civil; Electricidad y Electrónica; Energía y Agua; Fabricación Mecánica; Imagen y Sonido; Industrias Extractivas; Informática y Comunicaciones; Instalación y Mantenimiento; Madera, Muebles y Corcho; Marítimo-Pesquera; Seguridad y Medio Ambiente; Transporte y Mantenimiento; y Vidrio y Cerámica) y tienen presencia en otras. Una cifra muy superior si se compara con las cinco familias profesionales que están feminizadas (Administración y Gestión; Imagen Personal; Sanidad; Servicios Socioculturales y a la Comunidad; y Textil, Confección y Piel). Lo que pone de manifiesto una segregación horizontal y una clara brecha de género en las vocaciones.

En los datos señalados se incluye la modalidad de FPD, no obstante hay que destacar que, en general, el número de estudiantes que siguen este tipo de programa es muy reducido. En el curso 2016/2017 se impartió en 795 centros y accedieron a la misma un total de 20.357 estudiantes, y aunque en el curso 2017/2018 se incrementaron estas cifras hasta alcanzar los 890 centros y un total de 22.616 estudiantes, de estos 13.921 eran hombres y 8.695 eran mujeres (un 38,44\%). En este sentido, la mayor proporción de alumnado matriculado en FPD con respecto al total de alumnado matriculado en régimen presencial por enseñanza y familia profesional, se observa en las familias masculinizadas (MEyFP, curso 2017/2018). En todo caso, existe una presencia mayoritaria de hombres en este tipo de formación, que son los que principalmente se están viendo beneficiados de sus ventajas.

\section{3.- La socialización de género y su intervención en la elección de los estudios de formación profesional}

Cabría cuestionarse cuáles son las razones que determinan esa concentración en determinadas familias profesionales, dependiendo de si se trata de hombres o mujeres. Pues bien, tanto unos como otras, antes de elegir los estudios de FP, han recibido una educación que debe de haber contribuido al desarrollo de una serie de capacidades que les debe de haber permitido conocer, comprender y respetar la igualdad de derechos y oportunidades de hombres y mujeres, así como rechazar los estereotipos de género, como creencias que son aceptadas por la mayoría en relación a cómo son y cómo deben actuar unos y otras, esto es, por el rol que se 
espera que desempeñen por la propia sociedad, que impone determinados valores, patrones y modelos de conducta según el sexo. Una educación en la que no solo intervienen los centros educativos, y, en particular, los profesores como principales transmisores de valores y principios, sino que también lo hace, como la LOE indica, la familia, como agente al que se le confiere un papel fundamental, y en la que están implicadas las Administraciones educativas y la sociedad en su conjunto ya que, como señala la exposición de motivos de la norma, debe «apoyar al sistema educativo y crear un entorno favorable para la formación personal a lo largo de toda la vida».

Sin embargo, aunque el resultado debería ser la libre elección de sus estudios al haber adquirido unas capacidades en educación primaria y en ESO que les lleven a ese tipo de decisión, lo cierto es que los alumnos eligen condicionados por los estereotipos de género. Unos estereotipos que no deberían aceptar si se hubiera cumplido el objetivo que en este aspecto se persigue en la ESO (art. 23 c) LOE), ni ser transmitidos por la sociedad si realmente apoyara el sistema educativo y, por tanto, sus principios, fines y objetivos. Es cierto que poco a poco se ha ido produciendo un cambio con respecto a los roles de género, de hecho la formación de las mujeres ha contribuido al mismo y ha facilitado su incorporación al mercado laboral, sus posibilidades de promocionar en el trabajo y su participación en ámbitos de la vida económica y social tradicionalmente ocupados por hombres. Pero, esto no significa que no sigan existiendo estereotipos de género en cuanto al trabajo que deben desarrollar hombres y mujeres, en función de los roles tradicionales que unos y otras desempeñan en la sociedad (Mosteiro y Porto, 2017: 153), que están presentes en el momento de adoptar la decisión de qué estudios van a cursar.

En este sentido, la socialización de género tiene una enorme influencia en las elecciones que se realizan (Eccles, 2007: 665-691), y en este caso se pone de manifiesto en esa decisión. Las preferencias de los estudiantes vienen marcadas por esos estereotipos de género que se transmiten en la sociedad. Así, los agentes socializadores, en concreto, familia, profesores, grupo de pares, medios de comunicación, van a influir en las opciones académicas de los alumnos, por el hecho de que han recibido de los mismos unos valores en los que están presentes dichos estereotipos que hacen que las expectativas que tienen hombres y mujeres en cuanto a su formación académica y su posterior incorporación al mercado de trabajo difieran en función de su género. Así, aunque la edad de acceso a los estudios de FP varía, en su mayoría son jóvenes y adoptan una decisión influenciada, consciente o inconscientemente por su entorno, sobre todo por las familias y por el profesorado (Sáinz et al., 2012: 235-249; Eccles, 2007: 665-691), como principales agentes de socialización (Perry y Pauletti, 2011: 61-74).

En muchos casos las familias animan a sus hijas e hijos a realizar estudios vinculados a los roles de género, es más, tienen unas expectativas académicas distintas de unas y otros; de hecho manifiestan y transmiten esos sesgos de género desde la infancia a través de sus opiniones, sus comportamientos y sus valoraciones, lo que sin duda va a repercutir en su elección, obteniendo como resultado que las chicas no suelan elegir «estudios vinculados a la tecnología, la informática o las ciencias 
físicas» (Sáinz y Meneses, 2018: 26). Los propios profesores, de manera inconsciente, pueden trasmitir valores y estereotipos a los alumnos y a las alumnas, ya que pueden tener diferente expectativas de unos y de otras, y considerar que ellos son mejores en unas materias y ellas en otras (Sáinz et al, 2012; Sáinz y Meneses, 2018: 28); lo que en muchos casos supone orientar a las chicas para que realicen estudios tradicionalmente vinculados a la mujer. De ese modo pueden condicionar su elección, más aún en los casos en los que opinan sobre qué estudios son mejores para ellas y para ellos y, por tanto, qué profesión sería más adecuada (Sáinz y Meneses, 2018).

En la práctica, esto supone que persista y se transmita una imagen estereotipada de las profesiones, de forma que, en muchas ocasiones, se siga manteniendo que los hombres están más capacitados para realizar labores vinculadas a la ciencia, a la tecnología, a las matemáticas; lo que explica que las mujeres no suelan elegir estudios de este tipo y conecta con la falta de vocaciones STEAM. En definitiva, el hecho de que los adultos transmitan a niños y adolescentes una serie de creencias basadas en estereotipos de género sobre las distintas capacidades de los hombres y las mujeres, habitualmente viene a condicionar la elección de sus estudios (KurtzCostes et al., 2014: 603-618).

Junto a las familias y a los profesores, el grupo de pares también es muy importante en esa etapa (Sáinz et al., 2011: 401-411), ya que su elección puede suponer la aceptación o no por el mismo; de ahí que en la mayoría de los casos la elección que se hace busque esa aceptación y, por tanto, de este modo esté condicionada. Igualmente ejercen influencia los medios de comunicación (Sáinz, 2014: 61-88), que transmiten esos estereotipos de género, que definen las características de hombres y mujeres, los roles que normalmente adoptan y, por tanto, quienes deben dedicarse o no a determinadas profesiones.

En resumen, todos estos agentes ejercen una enorme influencia. En muchas ocasiones lo hacen reforzando esos estereotipos de género $\mathrm{y}$, por tanto, obteniendo el resultado contrario al deseado por el legislador, y ello a pesar de que en la exposición de motivos de la LOE se destaque el papel fundamental que tienen algunos de esos agentes de socialización, sobre todo las familias y los profesores, como «complemento necesario para asegurar una educación de calidad con equidad». Equidad que debe garantizar la igualdad de oportunidades de los alumnos y alumnas. El problema es que esos estereotipos de género están presentes en la sociedad y se ponen de manifiesto en el sistema educativo; «la escuela es un reflejo de la sociedad en la que vivimos» (González, 2009: 111). Se confiere a las familias y a los profesores un papel fundamental, pero no hay que olvidar que forman parte de una sociedad con unos patrones y modelos de conducta según el sexo, que impone sanciones por no desempeñar el rol de género previamente establecido, como la desaprobación (Mosteiro y Porto, 2017:154) que en gran parte de los casos obtienen las alumnas y los alumnos, sobre todo de sus familias y de su entorno, cuando se plantean elegir estudios que se consideran que les permitirán ejercer una profesión que se cree que no es propia de su género; lo que les lleva en muchos supuestos a seguir los estereotipos sociales, reproduciendo y consolidando las estructuras sociales preestablecidas. 


\section{4.- Conexión entre inserción laboral y formación profesional}

Como se ha comprobado, los roles que han venido asumiendo las mujeres han determinado que, en muchos casos, opten por realizar unos estudios y no otros, lo que va a tener repercusiones a la hora de acceder al mercado de trabajo, operando como una especie de barrera en términos de inserción laboral.

\section{1.- Breve referencia a la igualdad de oportunidades entre hombres y mujeres en el acceso al empleo}

En la actualidad, se siguen planteando problemas en el acceso al empleo en igualdad de oportunidades entre hombres y mujeres, a pesar de que el legislador lo haya previsto expresamente y no sea posible establecer perfiles profesionales en los que se incluyan factores que pueden ser considerados discriminatorios de forma directa o indirecta por razón de sexo (art. 17.1 Real Decreto Legislativo 2/2015, de 23 de octubre, por el que se aprueba el texto refundido de la Ley del Estatuto de los Trabajadores (ET); art. 5 de la $\mathrm{LOI}^{4}$; art. 35 Real Decreto Legislativo 3/2015, de 23 de octubre, por el que se aprueba el texto refundido de la Ley de Empleo ${ }^{5}$ ). Si bien, hay que recordar que cabe la posibilidad de que la negociación colectiva establezca «medidas de acción positiva», con el fin de «favorecer el acceso de las mujeres a todas las profesiones», en concreto, « reservas y preferencias en las condiciones de contratación de modo que, en igualdad de condiciones de idoneidad, tengan preferencia para ser contratadas las personas del sexo menos representado en el grupo o categoría profesional de que se trate» (art. 17.4 ET); de forma que tanto mujeres como hombres puedan beneficiarse de este tipo de medidas, dependiendo de cuál sea el sexo menos representado. Y que, en todo caso, por ley pueden establecerse exclusiones, reservas y preferencias de contratación (art. 17.2 ET); además, el Gobierno puede «regular medidas de reserva, duración o preferencia en el empleo» y «otorgar subvenciones, desgravaciones y otras medidas para fomentar el empleo de grupos específicos de trabajadores que encuentren dificultades para acceder al empleo» (17.3 ET).

Cabe destacar que la LOI contempla expresamente los programas de mejora de la empleabilidad de las mujeres, que se dirigen a todos los niveles educativos (art. 42), con el fin de aumentar su participación en el mercado de trabajo y avanzar en igualdad, y hace referencia a la posibilidad de establecer, mediante la negociación colectiva, medidas de acción positiva con el fin de «favorecer el acceso de las mujeres al empleo y la aplicación efectiva del principio de igualdad de trato y no discriminación en las condiciones de trabajo entre hombres y mujeres» (art. 43). Sin olvidar la obligación de las empresas de respetar la igualdad de trato y de oportunidades en el ámbito laboral, adoptando medidas que han de negociar y acordar con los representantes de los trabajadores. En este sentido, hay que resaltar la relevancia que tienen los planes de igualdad de las empresas (arts. 45 a 49 ), una 
materia que ha sido modificada por el RDL 6/2019, reduciendo el número de trabajadores de las empresas que determinan que exista la obligación negociar planes de igualdad en las mismas, situándolo en 50 o más trabajadores (art. 45.2 LOI, si bien, como establece en la disposición transitoria décima segunda LOI, se aplicará progresivamente), lo que es importante a efectos de adoptar medidas al efecto, en concreto, en relación a los procesos de selección y contratación, así como a la infrarrepresentación femenina en dichas empresas (art. 46.2 LOI).

Los planes de igualdad pueden ser una herramienta eficaz para combatir la segregación laboral y promover la ocupación de las mujeres en puestos para los que se precisan estudios de FP en los que están infrarrepresentadas, como se puede constatar en la práctica si se consideran las medidas que se contemplan en los de algunas de las empresas que «destacan por la aplicación de políticas de igualdad de trato y de oportunidades con sus trabajadores y trabajadoras», es decir, con distintivo de igualdad (art. 50 LOI) ${ }^{6}$. En concreto, en el plan de Aqualia se contienen determinadas prescripciones destinadas a promover el acceso de mujeres a puestos de trabajo masculinizados, para lo que se propone la colaboración a partir de acuerdos con organismos de formación, entre ellos los centros de FP, con el fin de que se formen mujeres que, posteriormente, se puedan incorporar a dichos puestos ${ }^{7}$. Por su parte, en el plan de Saint-Gobain Placo también se hace referencia expresa a la colaboración con escuelas de FP para favorecer el acceso a la empresa de mujeres con formación técnica; al igual que se contempla el reclutamiento en los centros de FP de personas candidatas del sexo menos representado en el puesto a cubrir ${ }^{8}$. En el plan de Aigües de Barcelona, Empresa Metropolitana de Gestió del Cicle Integral de L'Aigua, también se menciona el establecimiento de convenios de colaboración con centros de FP para la incorporación en prácticas de estudiantes mujeres con el perfil académico necesario para ocupar puestos de operarias para integrarlas en plantilla cuando haya una vacante' .

Unas medidas que pueden ser eficaces, pero el problema es que no son habituales. Lo más común es que los planes de igualdad contengan declaraciones genéricas, que carecen de operatividad para luchar contra los estereotipos de género que determinan que la mujer quede al margen en el acceso a determinados puestos de trabajo. No obstante, tras la entrada en vigor del Real Decreto 901/2020, de 13 de octubre, por el que se regulan los planes de igualdad y su registro y se modifica el Real Decreto 713/2010, de 28 de mayo, sobre registro y depósito de convenios

6 Real Decreto 1615/2009, de 26 de octubre, por el que se regula la concesión y utilización del distintivo «Igualdad en la Empresa».

7 Objetivo 2: Facilitar la incorporación del género subrepresentado a puestos en los que exista un desequilibrio manifiesto o una segregación ocupacional. Medida 2.1 del Plan de Igualdad de Aqualia (2015-2018), en http://www.igualdadenlaempresa.es/redEmpresas/distintivo/docs/Aqualia_PdI. pdf. En el mismo sentido, área 3 del plan de igualdad de Academia de Desarrollo Formativo (20162019), http:/ / www.igualdadenlaempresa.es/redEmpresas/distintivo/docs/ADF_PdI.pdf.

8 Apartado 6.1.2.h) II plan de igualdad de Saint Gobain Placo Ibérica (2019-2022), http://www. igualdadenlaempresa.es/redEmpresas/distintivo/docs/Saint_Gobain_Placo_PdI.pdf

9 Ámbito 3, acción 3.5 del Anexo III (Plan de Igualdad) del I convenio colectivo de Aigües de Barcelona, Empresa Metropolitana de Gestió del Cicle Integral de l'Aigua, S.A. para los años 2017-2023 (BOPB 13 junio 2017). 
y acuerdos de trabajo (RD 901/2020), esta situación debe cambiar. Precisamente, el art. 7.4 de esta norma establece que si el diagnóstico ${ }^{10}$ pone de manifiesto la infrarrepresentación de personas de un determinado sexo en ciertos puestos o niveles jerárquicos, el plan de igualdad necesariamente debe incluir medidas para corregirla; en concreto, se pueden establecer medidas de acción positiva con el fin de erradicar la segregación ocupacional por razón de género, tanto vertical como horizontal ${ }^{11}$.

\section{2.- La interdependencia entre estudios cursados e inserción laboral}

Es cierto que hombres y mujeres realizan unos estudios que les van a permitir acceder al mercado de trabajo, pero la elección de estos va a condicionar su inserción en el mismo, de forma que los roles que se ponen de manifiesto en la elección de estudios, después se proyectan en el mercado de trabajo. De este modo, la socialización de género supone un claro impedimento en términos de desarrollo profesional de las mujeres (Heilman, 2015). En muchos supuestos se parte del hecho de que romper la norma social a estos efectos puede suponer mayores dificultades para acceder al mercado de trabajo en un sector que puede estar feminizado o masculinizado (Emakunde, 2016: 80), razón por la que lo habitual es seguir esa norma social, evitando así el rechazo que puede suponer la ruptura en este caso no solo en el ámbito de relación habitual, sino también en el de la empresa.

Por tanto, aunque se debería partir de una posición de igualdad de hombre y mujer, en la que lo importante fueran sus competencias, sus habilidades individuales, la socialización de género supone un claro obstáculo para ello. El sujeto puede considerar que realiza su elección en libertad, pero no es así en la medida en que los agentes socializadores intervienen impactando en la decisión, a partir de los estereotipos de género que hacen que, por el hecho de ser hombre o mujer, se le atribuyan, sin tener en cuenta su consideración como individuo, solo por pertenecer al grupo, determinadas destrezas, o ciertas habilidades y expectativas, ligadas a opciones laborales en las que en realidad se percibe, en el caso de las mujeres, que existe una prolongación de las tareas reproductivas, domésticas y de cuidados como la atención a los dependientes (infancia, vejez); mientras que en el caso de los hombres se vincula a funciones de producción.

El hecho de que los estereotipos de género se interioricen, y no se cuestionen, conlleva que se vean limitadas las opciones a la hora de elegir los estudios y las posibilidades en el momento de acceder al mercado laboral, sobre todo en el caso de las mujeres que estudian FP, ya que los hombres, al tener una presencia más amplia en estos estudios, incluso en las familias feminizadas, acceden al empleo en un número de sectores más amplio. Esto determina que la segregación de género se reproduzca en el citado mercado, más aun si se tienen en cuenta las profesiones en

10 Art. 46.2 LOI y art. 7 RD 901/2020.

11 Los planes de igualdad que estén vigentes en el momento de su entrada en vigor deben «adaptarse en el plazo previsto para su revisión y, en todo caso, en un plazo máximo de doce meses contados a partir de la entrada en vigor de este real decreto, previo proceso negociador» (DT única RD 901/2020). 
las que existe una importante demanda de empleo y el hecho de que las mujeres no puedan acceder a las mismas porque no han cursado los estudios de FP vinculados a esas profesiones. Así, la segregación horizontal se proyecta en el mercado laboral, que, a su vez, suele estar vinculada a una segregación vertical por el hecho de que, aunque las mujeres pueden ser mayoría en un sector, son los hombres los que en muchos casos ocupan los puestos de mayor responsabilidad. En resumen, se puede apreciar como en el ámbito laboral se produce una división sexual del trabajo, distribuyendo las tareas entre hombres y mujeres en función de los roles de género y manifestándose de esa forma las expectativas que en cada caso tiene la sociedad de hombres y mujeres a partir de esa socialización de género.

\section{3.- Los efectos perjudiciales de la socialización de género para las estudiantes de formación profesional}

Existe una creciente empleabilidad de quienes estudian FP, si bien, a pesar de que en un $50 \%$ de las ofertas de empleo solo se hace referencia a estudios de FP, sin especificar, en el resto de ofertas las titulaciones más demandadas por familias profesionales son Administración y Gestión (13,31\%); Electricidad y Electrónica (7,76\%); Fabricación Mecánica (6,45\%); Informática y Comunicación (4,31\%); e Instalación y Mantenimiento (4,04\%). Cinco familias que concentran cerca del 36\% de las ofertas de empleo en las que se demanda candidatos con estudios de FP. El resto se distribuye entre las familias de Comercio y Marketing (3,06\%); Hostelería y Turismo (1,95\%); Transporte y Mantenimiento de Vehículos (1,71\%); Sanidad (1,49\%); y (con un 0,96\%) Artes Gráficas (Adecco Group, 2018: 126). Por sectores, destaca el Industrial; seguido de Hostelería y Turismo; e Informática. El resto de las ofertas se concentran en Construcción; Comercio; Metalurgia, Mineralurgia y Siderurgia; Automóvil, Maquinaria y Equipamiento Mecánico; Servicios, Mantenimiento; y Seguros (Adecco Group, 2018: 126 y 127).

Por ello, las mujeres ven reducidas sus posibilidades de inserción laboral. De ese $50 \%$ de ofertas de empleo específicas, tan solo el $15,69 \%$ se vincula a familias profesionales feminizadas. Así, aunque Administración y Gestión es la familia en la que se concentran el mayor número de ofertas (13,31\%), en el curso 2018/2019, el 37,6\% de quienes cursaron estudios de esta familia fueron hombres; por lo que la oferta también se dirige a una gran parte de alumnos. De hecho, de entre las familias profesionales que tienen una mayor concentración de alumnas, esta es la que acoge el menor número de ellas, seguida de Sanidad, en la que el número de alumnos se sitúa en un $27,6 \%$, si bien las ofertas alcanzan el 1,49\%, es decir, un número bastante reducido de ofertas a las que también acceden un porcentaje amplio de hombres. Ese número de alumnos disminuye en gran medida en Servicios Socioculturales y de la Comunidad, ya que su presencia es de un $12,9 \%$ en el citado curso, por lo que las ofertas de empleo prácticamente se dirigen a las mujeres, pero quedan reducidas al 0,39\%. El menor número de hombres con respecto a las familias profesionales que están más feminizadas se encuentra en la de Imagen Personal con un 0,7\% en el curso 2018/2019, aunque en este caso, de nuevo 
la oferta solo alcanza el 0,50\%. En todas estas familias se aprecia que las ofertas de empleo han disminuido en el año 2018 con respecto a las del año 2017, mientras que las vinculadas a familias profesionales como Instalación y Mantenimiento; Artes Gráficas y Química se han incrementado ligeramente (Adecco Group, 2018: 128). Así, si se acude a las cifras de desempleados que han cursado estudios de FP (con independencia de su modalidad, tal y como se contempla por el SEPE ${ }^{12}$ ) se puede observar cómo tanto en el caso de estudios de FPGM como de FPGS el número de mujeres desempleadas es superior al de hombres, en concreto, el porcentaje de desempleadas es del 65,14\% (de 230.483) y del 64,19\% (de 205.412), respectivamente (Observatorio de las Ocupaciones, 2019).

El problema es que el hecho de haber realizado determinados estudios condiciona su inserción laboral en un sector u otro, lo que supone que, mayoritariamente, las mujeres pasarán a realizar su labor en el sector servicios, mientras que los hombres lo harán en el sector industrial. Lo que tiene un efecto negativo para la mujer, sobre todo si se consideran los datos de los jóvenes desempleados según la actividad económica en la que han venido desarrollando su labor previamente a su última inscripción como demandantes de empleo. Así, el 63,4\% de jóvenes parados se incluye en el sector servicios, mientras que los desempleados en el sector industrial constituyen el 5,56\% (Observatorio de las Ocupaciones, 2019).

Por tanto, el hecho de que haya más mujeres desempleadas que hombres con estudios de FP en ambos casos, puede estar directamente relacionado, entre otros factores, con la ausencia de mujeres con una formación específica para desarrollar profesiones en el sector industrial, como, por ejemplo, sucede en el caso del metal o de la construcción, pero también en profesiones relacionadas con la tecnología, y, en concreto, con la informática. Así, la mujer queda desplazada de determinados nichos de empleo, de sectores vinculados a la industria en los que los salarios son más elevados, y en los que está el futuro. En este sentido, se puede observar cómo la brecha de género se vincula a la brecha salarial. Con un mismo nivel de formación, las mujeres perciben menores salarios que los hombres (INE, 2016; MEyFP, 2019c: 54), lo que obedece a factores como «la mayor incidencia de la parcialidad entre las mujeres, la mayor presencia femenina en sectores con menores retribuciones o la disrupción de las carreras profesionales de las mujeres» (MTMSS, 2019: 30; Haasler, 2014: 355 y 358). Sin embargo, también se puede constatar como en el caso de las ocupaciones paritarias las diferencias salariales entre hombres y mujeres se reducen considerablemente (INE, 2016; Fundación BBVA, 2018). Por tanto, la segregación ocupacional tiene consecuencias negativas desde el punto de vista económico, que se proyectan en la cuantía de las prestaciones por desempleo, o de las pensiones de jubilación (MTMSS, 2019: 41), es decir, más allá de su vida laboral; pero también social, sobre todo porque puede influir en decisiones tales como quién se quedará al cuidado de los hijos, o quién reducirá su jornada de trabajo, o

12 En cuanto a la dificultad para obtener datos relativos a FP Dual, vid. López, L.M.: «no hemos conseguido conocer de manera rigurosa datos sobre la FP Dual, que permitan, por un lado, comparar su tasa de inserción y éxito con la FP convencional y, por otro, conocer sus debilidades y oportunidades» (UGT, 2019: 6). 
solicitará una excedencia; lo que determina que se mantenga la situación de desigualdad de la mujer.

\section{5.- Soluciones y propuestas}

Las medidas adoptadas por el legislador para garantizar la efectividad de la igualdad de trato y de oportunidades entre mujeres y hombres en el mercado de trabajo pueden resultar insuficientes (Ballester, 2019: 38), pero, aunque el legislador contemple medidas para favorecer el acceso de las mujeres al empleo, estas no podrán desempeñar determinadas profesiones si no han adquirido la formación necesaria para ello. Por tanto, la solución no solo consiste en la aplicación de las medidas previstas en la normativa laboral para el acceso al empleo con el fin de combatir la segregación de género en las ocupaciones, sino que, además, es preciso anticiparse y abordar el problema con carácter previo al momento de la inserción de la mujer en el mercado laboral. Es fundamental que las mujeres se incorporen a los estudios tradicionalmente atribuidos a los hombres para que pueda mejorar su situación en el mercado de trabajo, accediendo a puestos que ahora solo ocupan estos. En este sentido, ya se vienen realizando diferentes actuaciones para que estas se integren en ciclos formativos masculinizados, en su mayoría vinculados al sector industrial y escasamente conocidos por las jóvenes, ya que no suelen contar con referentes de mujeres en este ámbito.

En concreto, hay que destacar algunas de las campañas de sensibilización y concienciación que se están llevando a cabo, como la de Hetel (Asociación de Centros de FP de la red concertada de Euskadi), con el lema «Pioneras de la FP», «Crea tu propio camino. Escoge $\mathrm{FP}^{13}{ }^{13}$, en las que se muestra el trabajo que realizan otras mujeres en la industria; un trabajo con amplia demanda y mejor remunerado que muchos de los puestos que están feminizados. O la campaña \#yotambienconstruyo ${ }^{14}$ lanzada por la Fundación Laboral de la Construcción, para dar visibilidad a las mujeres en este sector, e incrementar su participación en el mismo, que, a su vez, lidera el proyecto europeo, Women can Built ${ }^{15}$, en el que destacan las medidas dirigidas a la sensibilización y a la formación en materia de igualdad. Campañas que son positivas y que deberían servir de ejemplo para extenderse a otros ámbitos.

Además, en la Comunidad Valenciana se vienen convocando becas para fomentar el acceso de las mujeres a determinados ciclos formativos vinculados al sector industrial en centros educativos públicos valencianos ${ }^{16}$. En la misma línea, en Cataluña se promueve la matriculación de mujeres en ciclos industriales de grado superior con una bonificación de un $50 \%$ sobre el precio de la matrícula. Esto ha determinado que algunas mujeres hayan optado por cursar estudios de este tipo,

13 Vid. https: / / www.youtube.com/watch?v=m1jFcbIqnlw

14 Vid. https: / / www.youtube.com/watch?v=aX2ZCEE7eig

15 Vid. https:/ / www.womencanbuild.eu/

16 Vid., por ejemplo, Resolución de 31 de octubre de 2019, de la Dirección General de Formación Profesional y Enseñanzas de Régimen Especial, por la que se adjudican las subvenciones convocadas por la Resolución de 10 de abril de 2019, de la Consejería de Educación, Investigación, Cultura y Deporte (DOGV 15 noviembre 2019). 
por lo que debería adoptarse en otras Comunidades Autónomas, pero, a pesar de ello, en la Comunidad Valencia, algunos de los Grados no han recibido ninguna solicitud; en este sentido, su eficacia es limitada.

Por su parte, el V Plan Vasco de Formación Profesional 2018-2021 recoge entre sus principios la promoción de la igualdad entre hombres y mujeres, con el fin de «remover los obstáculos existentes que impiden que mujeres y hombres desarrollen sus trayectorias formativas y profesionales en igualdad y diversidad de opciones», y, en concreto, su objetivo 7 hace referencia a la necesidad de "promocionar la igualdad de mujeres y hombres en los centros de Formación». En este sentido, centra su actuación en la labor de los centros de FP y en el desarrollo de alianzas entre estos y las empresas con el fin de aumentar «la contratación de mujeres en los sectores estratégicos», lo que puede ser positivo, pero el problema no está tanto en el hecho de que no se contrate a las mujeres, sino en la propia elección de los estudios, y, previamente, en la infancia en la que está presente esa socialización de género; momento en el que hay que actuar.

El PEFP señala que «reducir la brecha de género existente en el acceso a determinados perfiles profesionales» es «una responsabilidad compartida por toda la ciudadanía, pero que nos afecta específicamente a las administraciones y a los interlocutores sociales», destacando como medida la «información y orientación profesional de calidad sobre los sectores profesionales y las ocupaciones». A estos efectos, cabe subrayar la importancia de la formación permanente del profesorado, como derecho y obligación de este colectivo, y responsabilidad de las Administraciones educativas y de los centros, en particular, en materia de igualdad entre hombres y mujeres (art. 102.1 y 2 de la LOE). La formación es esencial, en primer lugar, para que los profesores sean conscientes de los estereotipos de género y de los problemas que se generan, ya que forman parte de una sociedad en la que están presentes y puede que los hayan interiorizado, de hecho se encuentran en un sector feminizado, sobre todo en educación infantil y educación primaria (en el curso 2017/2018, 292.504 mujeres, y 57.585 hombres) y, en menor medida, en ESO, bachilleratos y FP (en dicho curso, 168.927 mujeres, y 119.620 hombres, según las Estadísticas del profesorado y otro personal del MEyFP), lo que supone que las mujeres son un agente esencial en la ruptura de las barreras en este ámbito, pero han de ser conscientes de las mismas y estar dispuestas a eliminarlas. Y, en segundo lugar, para conocer las posibles soluciones, medidas y buenas prácticas que han de adoptar.

En este sentido, entre los principios generales a los que hace referencia el art. 22 LOE, en relación a la etapa de ESO, destaca la necesidad de prestar «especial atención a la orientación educativa y profesional del alumnado». Y es el profesorado el que tiene encomendada «la orientación educativa, académica y profesional de los alumnos, en colaboración, en su caso, con los servicios o departamentos especializados» (art. 91.1 d) LOE). De ahí, la importancia de la formación a estos efectos y la necesidad de concretar su contenido, teniendo en cuenta en todo caso la igualdad de oportunidades entre hombres y mujeres. Esta labor es fundamental, con el fin de transmitir información a los alumnos y a las alumnas en relación a las posibilidades 
que existen en cuanto a los estudios de FP y en términos de inserción laboral, al margen los estereotipos de género.

Pero, no hay que olvidar que la familia es uno de los principales agentes de socialización, por eso es preciso actuar en este ámbito desde la infancia, para tratar de romper desde el inicio los estereotipos de género. En este sentido, hay que destacar el Plan de Coeducación 2017-2021 para los centros y las comunidades educativas de Navarra que podría adoptarse como referente en las distintas Comunidades Autónomas, sobre todo, porque se centra en desarrollar un itinerario de aprendizaje en materia de igualdad para su aplicación en las aulas en todas las etapas educativas (Programa SKOLAE Berdin bidean Creciendo en Igualdad), que también llega a las familias y al entorno más próximo al alumnado de todas las edades, y en la adquisición progresiva de las competencias básicas que van a permitir que las niñas y los niños puedan elegir y decidir sobre su futuro en libertad, sin condicionantes de género, a partir del conocimiento, identificando las desigualdades, y luchando contra ellas, dando visibilidad a las mujeres y a sus aportaciones.

Por tanto, las campañas, las ayudas a las jóvenes, las acciones de fomento de la igualdad en los centros de FP, la colaboración entre estos y las empresas, la formación al profesorado, el tratar de aumentar la contratación de mujeres en determinados sectores, pueden ser positivas, pero para solucionar el problema es preciso ir más allá, anticiparse, actuar en todas las etapas educativas, llegar a las familias, implicarlas, hacerlas partícipes de actividades en los centros en las que se aborden desde la infancia la igualdad entre hombres y mujeres. Si solo se actúa en el momento de acceder al mercado de trabajo, o en el de la elección de los estudios, implicando únicamente a las alumnas, lo más probable, como sucede, es que la intervención sea positiva pero no tenga el éxito esperado.

\section{Bibliografía}

ALEMÁN, Francisco (2018). «El empleo y el emprendimiento juvenil y sus tribulaciones estructurales: notas críticas al hilo de la obra «el emprendimiento para fomentar y promover la inclusión social y laboral de la juventud»» en Revista Española de Derecho del Trabajo, 206 (Aranzadi Instituciones, BIB 2018\6614).

ADECCO GROUP (2018). Informe infoempleo Adecco. Disponible en: https:/ /www. infoempleo.com/informe-infoempleo-adecco/ (Fecha de consulta: 23/01/2020).

BALLESTER, María Amparo (2019). «El RDL 6/2019 para la garantía de la igualdad de trato y de oportunidades entre mujeres y hombres en el empleo y la ocupación: Dios y el diablo en la tierra del sol» en Femeris, Vol. 4, No2, pp.14-38.

BUCHHOLZ, Sandra y Daniela GRUNOW (2006). «Women's Employment in West Germany» en BLOSSFELD, H.P. \& H. HOFMEISTER (Eds) (2006). Globalization, Uncertainty and Women's Careers. An International Comparison, Cheltenham: Edward Elgar. 
ECCLES, Jacquelynne S. (2007). «Families, schools, and developing achievementrelated motivations and engagement» en GRUSEC, J. E. y P.D. HASTINGS (2007). Handbook of socialization. Theory and research, Nueva York: The Guilford Press.

EMAKUNDE (2016). La incidencia del valor de la igualdad en la elección de estudios de grado superior por alumnado de $2^{\circ}$ de Bachillerato en Euskadi, Vitoria-Gasteiz: EMAKUNDE - Instituto Vasco de la Mujer.

FUNDACIÓN BBVA (2018). Segregación de Género en las Ocupaciones, IVIE. Disponible en: https://www.fbbva.es/wp-content/uploads/2018/11/FBBVA_Esenciales_30.pdf (Fecha de consulta: 23/01/2020).

GOBIERNO DE NAVARRA. SKOLAE Berdin bidean. Creciendo en Igualdad. Plan de Coeducación 2017-2021. Disponible en: https://www.SKOLAE-Berdin-BideanCreciendo-en-Iguadad_Programa.pdf (Fecha de consulta: 02/01/2020).

GOBIERNO VASCO (2019). V Plan Vasco de Formación Profesional. Disponible en: https://www.euskadi.eus/contenidos/informacion/fpgeneral/es_def/ adjuntos/V-PLAN-FP-CASazk.pdf (Fecha de consulta: 02/01/2020).

GONZÁLEZ, Isabel (2009). «La orientación académica y profesional en clave de igualdad» en Revista Participación educativa, N¹1, pp.110-121.

HAASLER, Simone (2014). «The Impact of Learning on Women's Labour Market Transitions» en Research in Comparative and International Education, Vol. 9, No3. Disponible en: www.wwwords.co.uk/RCIE (Fecha de consulta: 02/01/2020).

HEILMAN, Madeline (2015). «Gender stereotypes: Impediments to women's career progress» en Welpe, I. et al. (Eds.) (2015), Auswhal von Männern und Frauen als Führungskräfte. Perspektiven aus Wirtschaft, Wissenschaft, Medien und Politik, pp.73-84.

INE (2016). Encuesta de Estructura Salarial Cuatrienal 2014. Disponible en: https:/ / ine.es /dyngs/INEbase/es / operacion.htm?c=Estadistica_C\&cid=1254736177 $025 \&$ menu $=$ resultados\&secc $=1254736195109 \& i d p=1254735976596$ (Fecha de consulta: $28 / 01 / 2020)$.

KURTZ-COSTES, Beth et al. (2014). «Gender and age differences in awareness and endorsement of gender stereotypes about academic abilities» en European Journal of Psychology of education, Vol. 29, N²4, pp. 603-618.

MEyFP (2019a). Panorama de la Educación, Indicadores de la OCDE 2019. Informe Español. Disponible en: https:/ / www.educacionyfp.gob.es/inee/dam/jcr:4689798f5c6b-4bab-b5fe-58ad7004c6fd/panorama\%20de\%20la\%20educación \%20 2019-línea-def.pdf, (Fecha de consulta: 28/01/2020).

MEyFP. I Plan Estratégico de Formación Profesional del Sistema Educativo 2019-2022. Disponible en: http://todofp.es/dam/jcr:163978c0-a214-471e-868d-82862b5a3aa3/plan-estrategico-fp.pdf (Fecha de consulta: 16/01/2020).

MEyFP. Estadísticas del alumnado de Formación Profesional, curso 2017/2018. Disponible en: http://www.educacionyfp.gob.es/dam/jcr:a461da71-8247-44429ab1-e0401fff8039/nota-17-18.pdf, (Fecha de consulta: 20/12/2019).

MEyFP. Estadísticas del profesorado y otro personal. Curso 2017/2018. Disponible en: https://www.educacionyfp.gob.es/servicios-al-ciudadano/estadisticas/nouniversitaria/profesorado/estadistica.html (Fecha de consulta: 22/01/2020). 
MEyFP (2018). Datos y cifras. Curso escolar 2018/2019. Disponible en: http:/ /www. educacionyfp.gob.es/dam/jcr:f17caadd-acf4-41e5-8143-8489ed4b0cf2/datosycifras1819esp.pdf (Fecha de consulta: 20/01/2020).

MEyFP (2019b). Estadística de las Enseñanzas no universitarias. Datos avance 20182019. 21 de agosto de 2019. Disponible en: https://www.educacionyfp.gob.es/ dam/jcr:c876531a-31c1-4f7b-ad19-f85e6d3b49e3/notres1819.pdf (Fecha de consulta: 02/01/2020).

MEyFP(2019c). Igualdad en cifras, Secretaría General Técnica. Disponible en: https:/ / sede.educacion.gob.es/publiventa/descarga.action?f_codigo_agc=19415 (Fecha de consulta: 28/01/2020).

MOSTEIRO, María Josefa y PORTO, Ana María. (2017). «Análisis de los estereotipos de género en alumnado de formación profesional: diferencias según sexo, edad y grado» en Revista de Investigación Educativa, Vol. 35, Nº1, pp. 151-165.

MTMSS (2019). Mujeres en el mercado de trabajo, mujeres pensionistas y mujeres migrantes en el siglo XXI. Disponible en: http://www.mitramiss.gob.es/es/destacados/HOME/documentos/Informe_mujeres_febrero_2019.pdf (Fecha de consulta: 02/01/ 2020).

OBSERVATORIO DE LAS OCUPACIONES. SEPE (2019). Informe del Mercado de Trabajo de las Mujeres Estatal. Datos 2018. Disponible en: https:/ /www.sepe.es/ HomeSepe/que-es-el-sepe/comunicacion-institucional/publicaciones/publicaciones-oficiales/listado-pub-mercado-trabajo/informe-mercadotrabajo-estatal-mujeres.html (Fecha de consulta: 19/12/2019).

OCDE (2019). Panorama de la educación 2019. Disponible en: https://sede.educacion.gob.es/publiventa/descarga.action?f_codigo_agc=19884 (Fecha de consulta: 03/01/2020).

PASTOR, Albert (2019). «Las medidas laborales del Real Decreto-Ley 6/2019, de 1 de marzo, de medidas urgentes para garantía de la igualdad de trato y de oportunidades entre mujeres y hombres en el empleo y la ocupación: un paso hacia la efectividad de la igualdad de trato y de oportunidades desde la corresponsabilidad» en IUSLabor 1. Disponible en: https://www.upf.edu/ documents / 3885005/224374284 / 4.Pastor.pdf/fc237263-43cf-12c8-b750-d3a88c2a0280 (Fecha de consulta: 19/12/2019).

PERRY, David y Rachel PAULETTI (2011). «Gender and adolescent development» en Journal of Research on Adolescence, Vol.21(1), pp. 61-74.

SÁINZ, Milagros (2014). «Sacando los pies del tiesto. Jóvenes y elección de estudios» en CASTAÑO, C. y J. WEBSTER, J. (eds.) (2014), Mujeres, ciencia y tecnologías de la información y la comunicación, Barcelona: Aresta, pp.61-88.

SÁINZ, Milagros et al. (2011). «Influencia de los padres y los profesores en la elección de estudios TIC», en CHIVITE, M. J., HERNÁNDEZ, M. B. y MONZÓN, M. E. Frontera y Género: en los límites de la multidisciplinariedad, Madrid: Plaza y Valdés, pp.401-411.

SÁINZ, Milagros, PÁLMEN, Rachel y Sara GARCÍA (2012). «Parental and secondary teachers' perceptions of ICT professionals, gender differences and their role in the choice of studies» en Sex Roles, 66, pp. 235-249. 
SÁINZ, Milagros y Julio MENESES (2018). «Brecha y sesgos de género en la elección de estudios y profesiones en la educación secundaria» en Panorama Social, $\mathrm{N}^{\circ} 27$, pp.23-31.

UGT (2019). Estudio sobre la situación y evaluación de la Formación Profesional Dual en la Comunidad de Madrid. Disponible en: https://madrid.ugt.org/sites/madrid. ugt.org/files/estudio_fpd_comunidad_de_madrid_revision_final_publicacion.pdf (Fecha de consulta: 20/01/2020).

Recibido el 23 de junio de 2020 Aceptado el 17 de marzo de 2021 BIBLID [1132-8231 (2021): 83-104] 